\title{
Automatic Inceptor Decoupling System in Electronically Coupled Active Sidesticks for Dual Pilot Helicopters
}

\author{
R. dos Santos Sampaio ${ }^{1}$ \\ German Aerospace Centre (DLR), Braunschweig, Niedersachsen, 38108, Germany
}

\begin{abstract}
The active inceptor system provides the ability to electronically couple the pilot's and copilot's inceptors in dual pilot helicopters. Also, a novel inceptor decoupling can be programmed in order to prioritize one pilot control station in case of inceptor obstruction or force fight between pilots. Through the measurement of the forces in real time, the inceptors are automatically decoupled if both pilots apply forces in opposing directions above the specified force threshold. The paper aims to investigate maximum force threshold for the automatic inceptor decoupling that still can be acceptable in terms of controllability. To this end, four test pilots participated in the experimental tests in a ground-based simulator featuring active inceptors in a dual pilot helicopter cabin. The safety severity envelope were developed and validated through takeover control tasks in low level flights. The maximum force threshold for automatic inceptor decoupling is found to be $30 \mathrm{~N}$ in pitch axis, i.e., that the maximum force threshold which control oscillations are linked at least to level 2 handling qualities. Additionally, an active force fading logic is implemented and showed to effectively reduce the control activity and helicopter attitude variation during the automatic inceptor decoupling.
\end{abstract}

\section{Nomenclature}

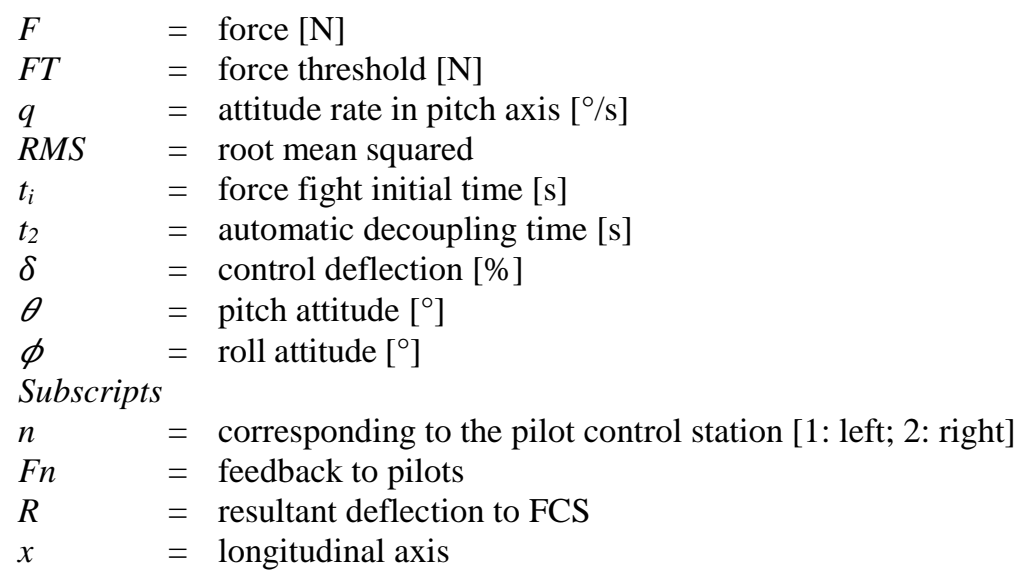

\section{Introduction}

The fly-by-wire (FBW) technology allows the total elimination of the complex mechanical linkages in the flight control system (FCS), since all commands and signals can be transmitted electrically through wires. Within this context, a remarkable feature of the state-of-the-art FCS is the introduction of coupled active inceptors, whereby the pilot's inceptors are electronically connected to the copilot's inceptors, as opposed to the traditional mechanical linkage across the cabin in dual pilot operations. The terminology inceptor indicates any device that is used to provide pilot's control inputs.

The active inceptor system (AIS) typically provides stick position as a function of the force sensed at the grip from an input applied by the pilot [1]. In the coupled active inceptors, the force deflection characteristics of a mechanical linkage can be emulated through the ability of the AIS to provide synthetic mass-spring-damper feel in real time

\footnotetext{
${ }^{1}$ Research Scientist, Test Pilot, PhD Candidate; Institute of Flight Systems, Helicopter Department (DLR).
} 
manner. The inceptor coupling is achieved by allocating dedicated electrical links to transmit the forces of one sidestick to the other. Thus, the behavior of the inceptors on the pilot flying's (PF) control station can be replicated on the inceptors of the pilot monitoring (PM) through the electronic coupling of the AIS.

The electronic inceptor coupling has proven to be advantageous compared to the uncoupled counterparts. For instance, the PF's response time (i.e., reaction time) to recognize control interference is reduced using coupled inceptors [2], [3]. Moreover, due to aircraft dynamics, the force feedback of the coupled AIS conveys information to the PM that a change has been commanded before the aircraft response to the PF's input [4], providing useful anticipatory responsiveness [5] and increased situational awareness [6].

Nonetheless, the pilots' inceptors permanently coupled across the cabin can produce safety problems. In case of control interference in one pilot station, the other control station is irreversibly affect, because both control stations are actually linked across the dual pilot cabin. If this interference occurs in typical helicopter scenarios of minimal safety margin (e.g., flights near obstacles), it might trigger oscillations in the helicopter attitude, which is an undesired effect in such scenarios. Unsurprisingly, there is a class of accidents classified as loss of control (LOC) that is caused by control interference (INT), which includes events of lack of positive control transfer between pilots and inadvertent control obstruction [7]. A recurrent case of LOC-INT is the unsuccessful takeover control maneuver performed by the flight instructor (FI) in training flights. When the trainee pilot does not recognize the action of the flight instructor, a force fight condition (two pilots applying force in different directions) can emerge, producing an unsafe state in low level flights.

The challenging outcome caused by the control interference during takeover maneuvers is illustrated in Fig. 1. The graphs refer to a flight condition described in one accident investigation report [7] and reproduced in a simulator for a flight training mission. The pilots are referred to as trainee pilot (initially the PF) and FI (the pilot that performs the takeover control due to a safety critical condition). At time $=0$, the FI counteracts the pitch down input of the trainee pilot. Due to the force feedback, the trainee pilot relinquishes control in less than two seconds (arrow 1). This action triggers an overshoot in the inceptor position, because the force to counteract the trainee pilot is now transferred to the deflection of the stick according to the force-deflection curve (arrow 2). The stepwise control input causes significant helicopter attitude variation with high attitude rate (arrows 4 and 3, respectively). In case of flight near obstacles, the helicopter motion can lead to marginal safety margins.
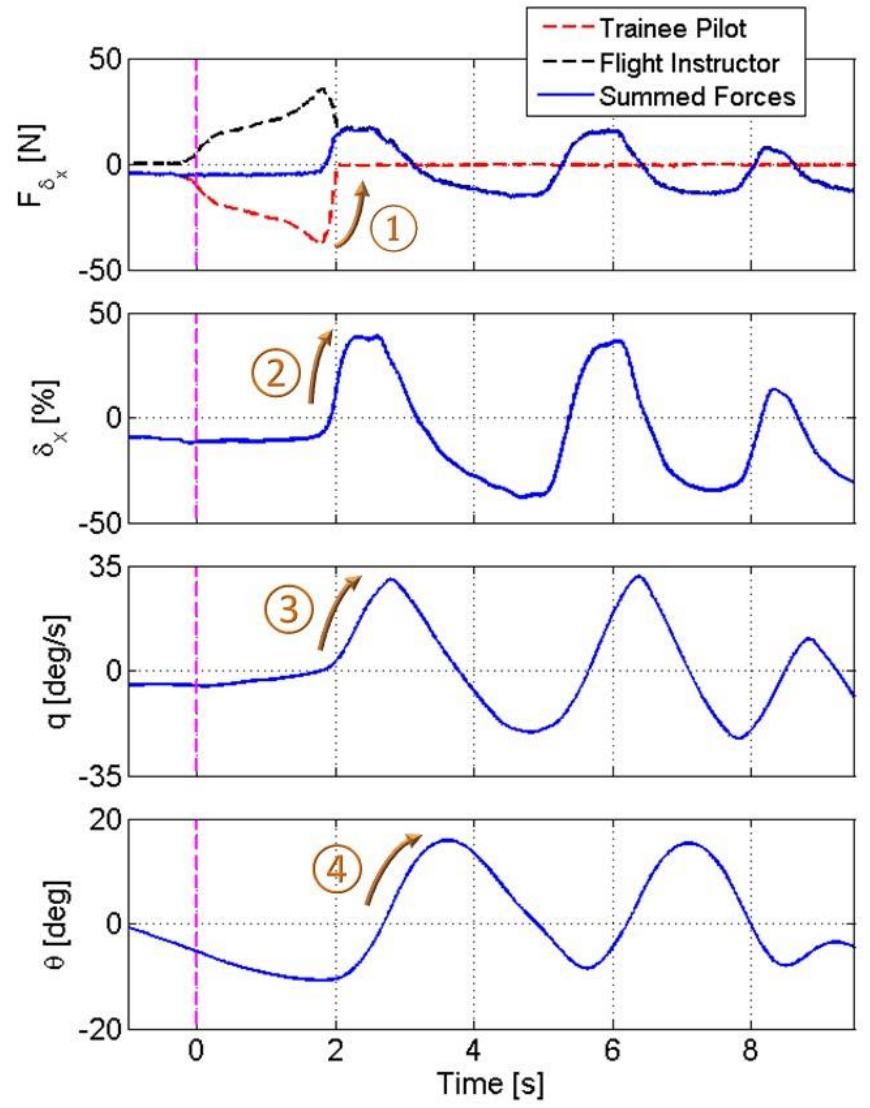

Fig. 1 Force fight during takeover control maneuver. 
Due to the ability to measure in real time the forces applied in the AIS, electronically coupled sticks can be programmed to automatic decouple inceptors in case of control interference above a certain force threshold (FT). When both pilots apply inputs at the same time and the force difference reaches a predetermined FT (e.g., $30 \mathrm{~N}$ ), the AIS can automatically deactivate one pilot control station in order to increase the control authority of the pilot who is performing the control takeover maneuver (e.g., deactivate the trainee pilot to prioritize the flight instructor). It should be noted that the pilots' attention in flights near the ground is generally directed outside the cabin, and some time might be necessary to understand control interventions, e.g., during takeover control maneuvers. The decoupling function can not only limit the magnitude of the force fight through the setting of the FT, but also reduce drastically the force fight time. Therefore, a decoupling function to prioritize one control station is a feasible solution in case of control interference. Besides the pilot assistance during takeover control maneuvers, an automatic inceptor decoupling system can be used to isolate malfunctions in one control assembly; disengage a control station in case of an incapacitated pilot; or switch off controls whilst transporting a passenger in the copilot seat.

As a safety relevant function, one of the goals of the automatic inceptor decoupling system is to perform takeover control with minimal consequence to the controllability. However, there remains a lack of evidence regarding the consequences of such system. If the FT is too high, the sudden decoupling while pilots are applying forces in opposing directions can lead to control discontinuities, stepwise inputs, and helicopter attitude transients. Given the scientific significance of this topic for FCS design in future FBW helicopters, this paper aims to investigate the maximum force threshold for the automatic inceptor decoupling function, which can be achieved by answering the following two research questions:

1) Which is the highest acceptable FT level in terms of controllability for automatic inceptor decoupling in case of control interference in low level flights?

2) Considering the control deflection and the helicopter attitude transients caused by the automatic inceptor decoupling, can these transients be alleviated by active inceptor functions?

\section{Coupled Active Sidestick System}

The pilot-inceptor-vehicle loop, including a simplified signal flow used to couple the active sidesticks, is shown in Fig. 2. The forces applied by pilots are measured by the force sensors and sent to a computer within the AIS, here represented by the prioritization logics block. The force-deflection characteristics (graphs of $F x \delta$ ) are adjusted according to the internal control laws, the forces on inceptors, and flight data from the helicopter dynamics. The information sent back to the sidestick units are indicated by the inceptor deflection $(\delta)$ in Fig. 2. The active modifications can include not only the inceptor position, but also all static and dynamic parameters, like natural frequency, damping ratio, stiffness, friction and others.

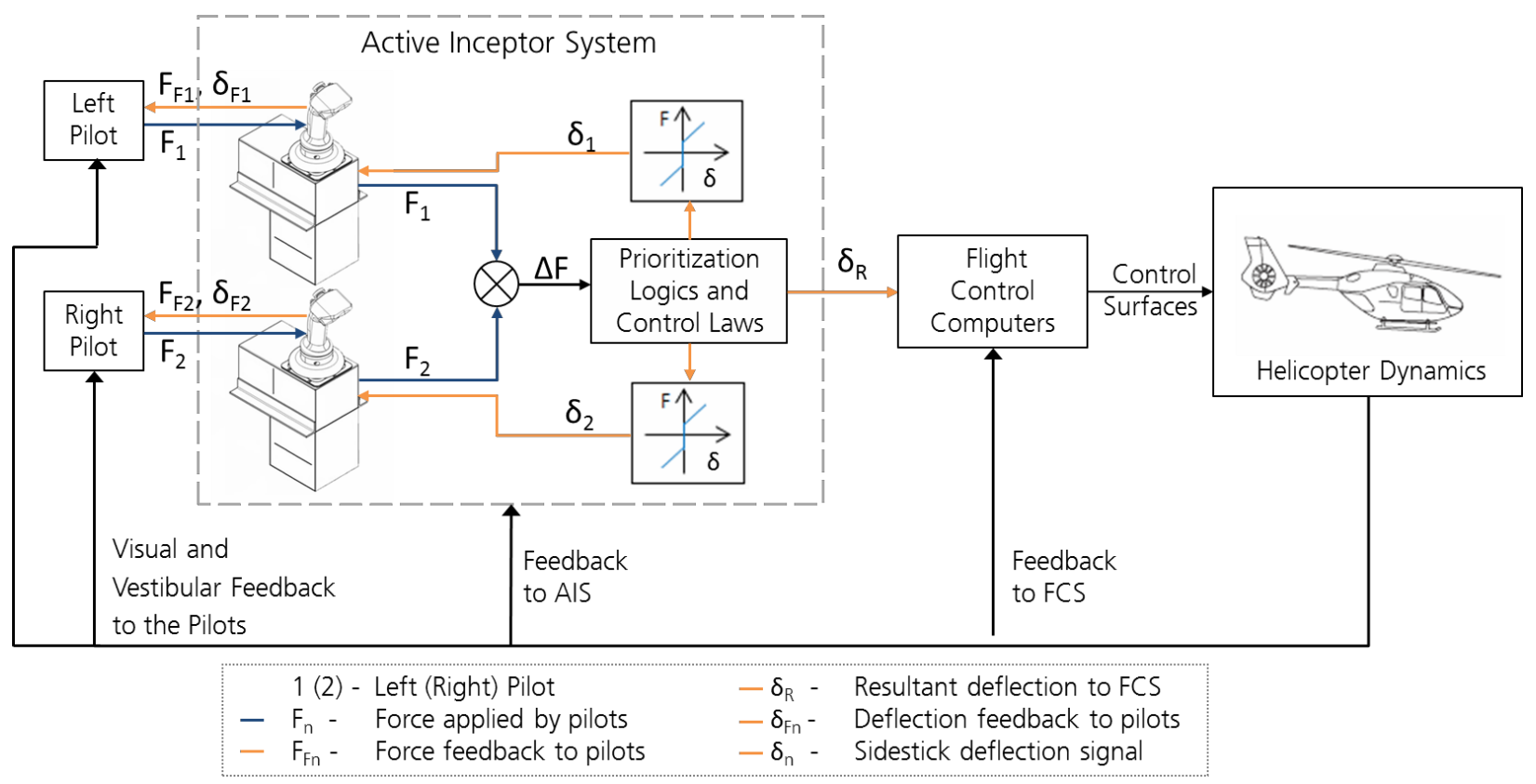

Fig. 2 Pilot-inceptor-aircraft loop including the active sidestick coupling. 
Pilots can feel the corresponding deflection of the sticks through the servo motor in each axis as a function of either the applied forces from the opposite sidestick, or the supplementary tactile cues generated by the AIS.

In case of emulation of the mechanical cross-cabin linkage, the sidestick units behave in the same way as if rigidly connected as the result of equal sidestick deflection output by the prioritization logics block $\left(\delta_{1}=\delta_{2}\right)$. Since both sidesticks are in the same position with respect to their neutral points, the input signal to the flight control computers $\left(\delta_{R}\right)$ will correspond to the actual position of the sidesticks $\left(\delta_{F 1}\right.$ and $\left.\delta_{F 2}\right)$.

The automatic decoupling function is activated through the information provided by the force sensors. In case of a force fight between pilots (i.e., they apply inputs in different directions), a given force threshold is specified whereby the inceptor coupling is disengaged if the opposing forces surpass this threshold. An important design consideration is the definition of which control station will retain full control authority (single pilot priority) and which one will have no control authority (deactivated pilot). In this paper, the functions are programmed to prioritize the control cabin of the right pilot, who is taking over control (hereafter FI). If the system detects the unsafe condition (two pilots applying inputs at the same time) and the force threshold is reached, the control station of the left pilot is deactivated (henceforth trainee pilot). Thus, the FI's authority is increased by removing the influence of the trainee pilot on the maneuver of control takeover.

Fig. 3 shows the trainee pilot initially flying the vehicle without any force input from the flight instructor. At time $t_{i}$, the FI starts to counteract the trainee. At time $t_{2}$, when the trainee pilot (acting as PF) is automatically deactivated, only the FI input is considered by the sidestick controller and the inceptor is deflected to the corresponding force applied by the FI (Fig. 3a). A likely overshoot in the control deflection in expected, because the force of one pilot is suddenly changed to zero, which causes a stepwise change in the FI's force. This condition is reflected in the summed control output, which will generate a corresponding control deflection, based on the force control mechanical characteristics (e.g. force-deflection curve) and control laws. Ultimately, the vehicle attitude variations can occur as a consequence of the control overshoots.

In order to compensate the opposing force after the decoupling is commanded by the system and to alleviate the residual oscillations in control deflection, a force fading logic, namely Counter Force, is implemented (Fig. 3b). The Counter Force function retains the force applied by the opposing pilot (in this case, the trainee pilot) at the moment of the decoupling $(t 2)$, and reduces this value gradually to zero in one second, providing the opportunity to the FI pilot to steadily alleviate the force during this period of time. The active force variation compensates the stepwise control caused by the sudden automatic inceptor decoupling and attenuates the adverse helicopter attitude oscillations.

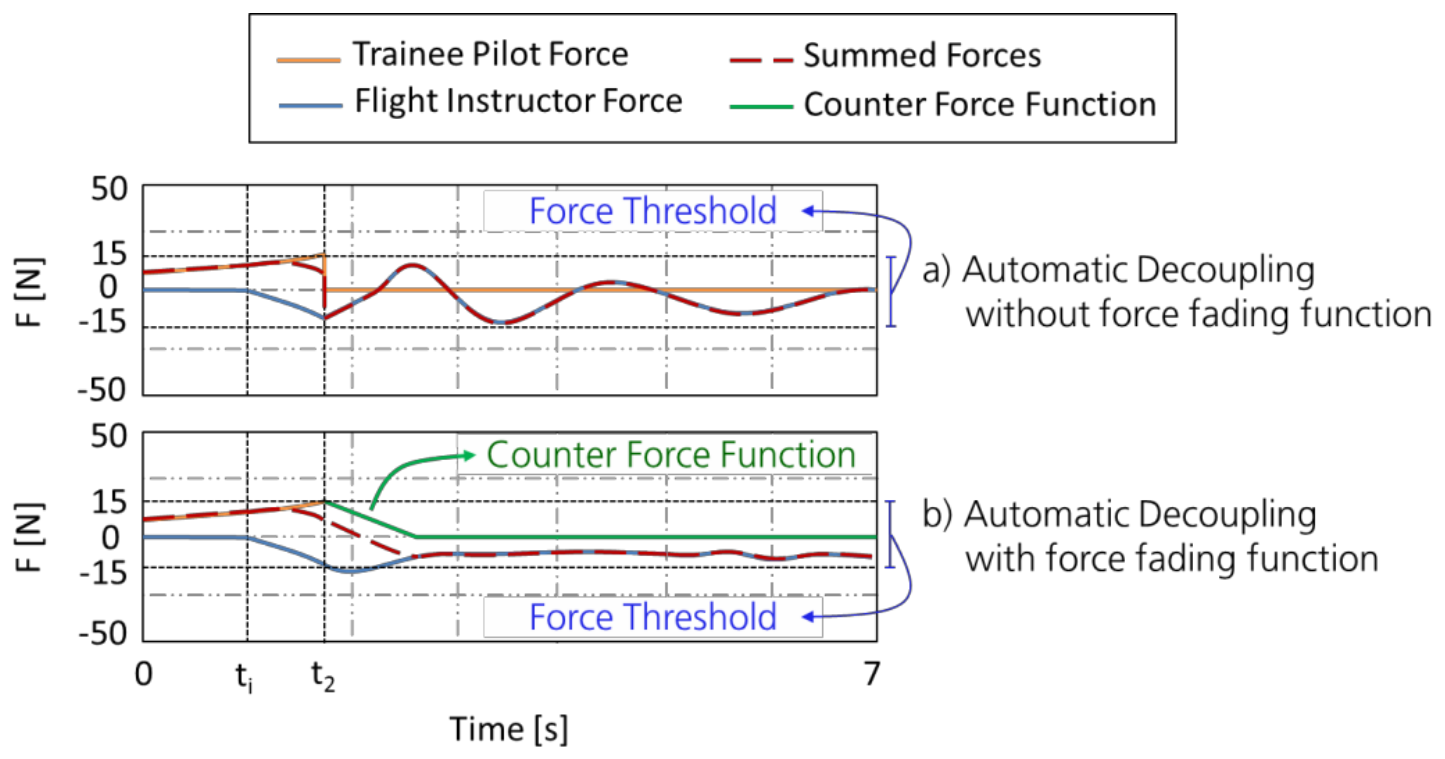

Fig. 3 Takeover control with and without force fading logic (Counter Force). 


\section{Evaluations}

\section{A. Experimental Setup}

The experiments were conducted in the "Dual Pilot Active Sidestick Demonstrator" (2PASD), a fixed-based simulation environment operated by the Institute of Flight Systems at the German Aerospace Centre - DLR (Fig. 4) [6]. The simulation platform features coupled sidesticks and operates in a pseudo real-time environment, using the program 2Simulate as the simulation framework [8]. The inceptor coupling is modeled by MATLAB/Simulink [9].

Two helicopter models are tested in the evaluations: the baseline and the modified helicopter. In both cases, the response type of the helicopter tested is attitude command attitude hold (ACAH) in pitch and roll axes, and rate command attitude hold (RCAH) in yaw and heave axes.

The baseline helicopter, which is detailed in [10], refers to the augmented full-envelope simulation model of the ACT/FHS (Active Control Technology /Flying Helicopter Simulator), a highly modified version of the EC 135 used by DLR as an in-flight simulator for research purposes [11]. For this reason, its performance and flying qualities do not reflect operational variants of the rotorcraft type.

A second helicopter model, namely modified helicopter, is examined. To this end, the baseline helicopter is modified to characterize a vehicle with degraded stability compared to the initial one. This approach provides the opportunity to analyze the system in conditions representative of handling qualities (HQ) level 3, as described by US Army Aeronautical Design Standard (ADS-33E) [12]. The goal is to verify if the automatic decoupling system can trigger control difficulties to pilots during high gain tasks in case of poor HQ. The modification of the helicopter stability was performed by reducing the values of the control response derivatives $\theta$ and $\phi$ by $30 \%$ compared to the nominal values of the baseline helicopter, and by adding $300 \mathrm{~ms}$ time delay in the flight control system.
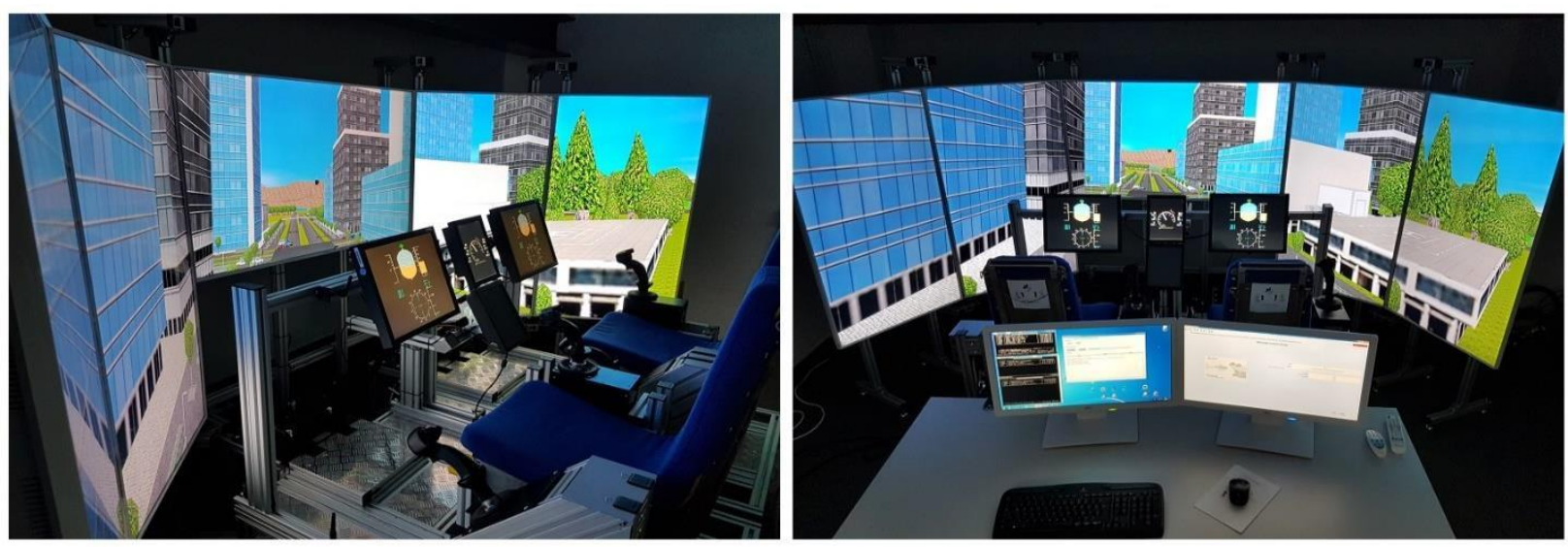

Fig. 4 Dual Pilot Active Sidestick Demonstrator - 2PASD.

\section{B. Methodology}

The two research questions are answered by gathering the subjective and objective data after the automatic inceptor decoupling in low level flights. Throughout the evaluation, the assessing pilots tested the system acting as a flight instructor. To this end, takeover control maneuvers by the FIs are employed with different FT. The assessing pilot awarded ratings using the decision-tree transient rating (TR) scale [13], from A to $\mathrm{H}$, according to the resulting effects in terms of helicopter attitude and control deflection transients after the automatic inceptor decoupling. The TR can be seen in the right column of Fig. 5 .

The classification of the transient effects is plotted against the objective data (i.e., control deflection and helicopter attitude variations) to define a severity envelope for the takeover control maneuver using the automatic inceptor decoupling, which is based on the severity classification of the integrated transient classification [14], i.e., the left column of Fig. 5. New tests are performed to validate the severity envelope and to answer the main research questions, regarding the maximum FT and the effectiveness of the active functions (i.e., Counter Force) to alleviate control overshoots and attitude oscillations.

\section{Sampling}

Four test pilots participated in the force threshold assessment. Whereas one test pilot from DLR developed the severity envelope (pilot A), three test pilots from the Bundeswehr Technical and Airworthiness Center for Aircraft 
(WTD 61) were invited to validate the envelope previously developed (pilots B to D). The flight experience of the test pilots is described in Table 1.

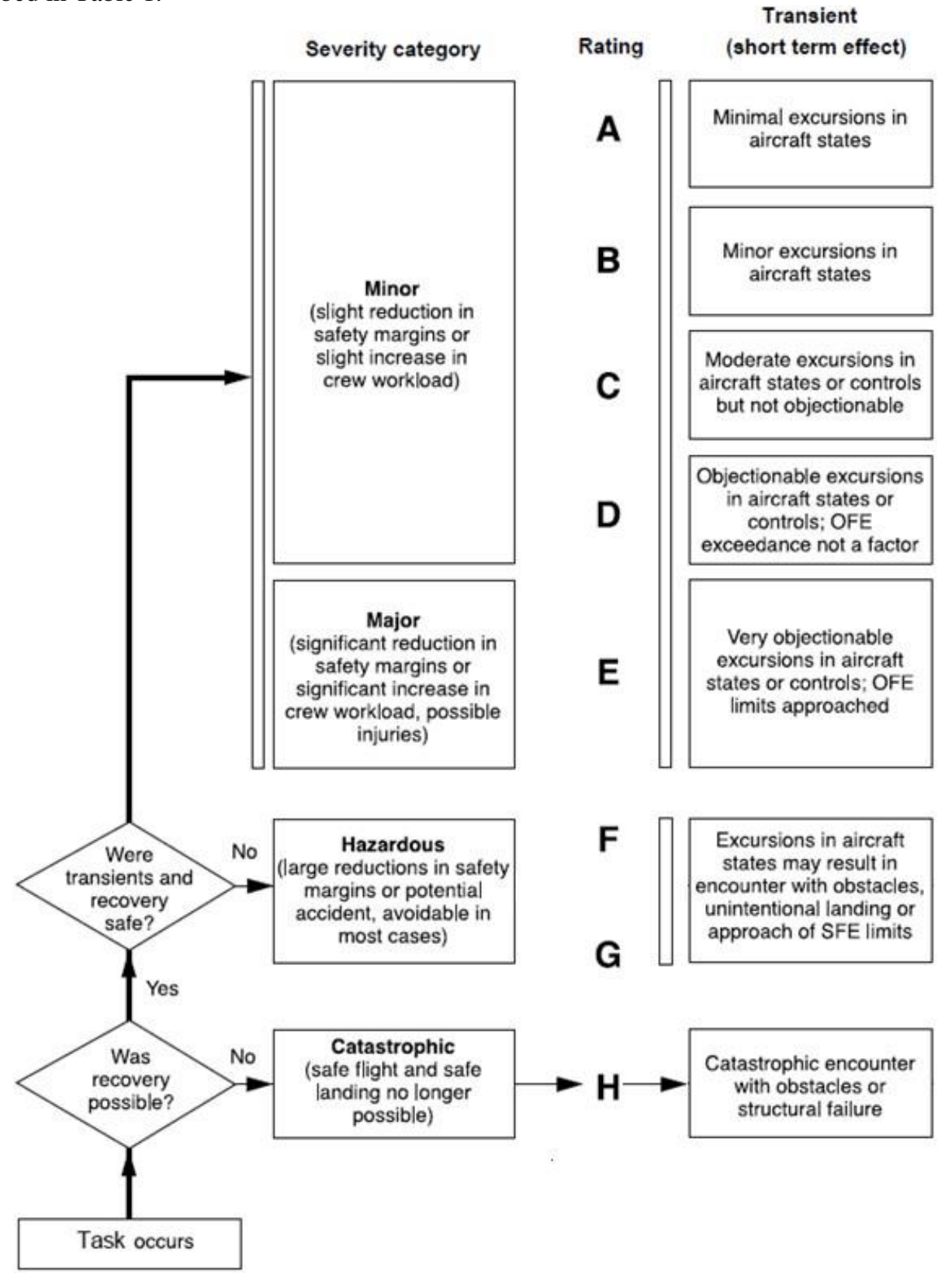

Fig. 5 Integrated Transient Classification [14]

Table 1 Pilots experience

\begin{tabular}{lcccc}
\hline Background & Pilot A & Pilot B & Pilot C & Pilot D \\
\hline Total Flight Hours & 1800 & 2300 & 5000 & 4200 \\
\hline Age [years] & 37 & 40 & 59 & 46 \\
\hline Test Pilot & Yes & Yes & Yes & Yes \\
\hline Flight Instructor & Yes & Yes & Yes & Yes \\
\hline Main Helicopter & AS-350; AS-355; & NH-90; CH-53; & NH-90; UH-1D/H; & CH-53; UH-1D/H; \\
Flown & UH-60 & UH-1D & WG-13 & UH-Tiger; H-145 \\
\hline
\end{tabular}




\section{Procedures}

A briefing containing the scope of the research and the system description was presented to the pilots, including the DLR test pilot who contributed as the trainee pilot. Each pilot flew at least three practice trials with automatic inceptor decoupling before each recorded test point. The automatic inceptor decoupling was tested in FT of $20 \mathrm{~N}$, $30 \mathrm{~N}$ and $40 \mathrm{~N}$ in pitch axis, as this axis was found to be more critical than the roll axis in terms of stability. Pilot A flew 90 test points for the development of the severity envelope for the automatic decoupling, and pilots B to D performed 36 test points for the validation phase.

\section{E. Task}

To evaluate the system, the task of transition to hover mission task element (MTE) was employed. The task consists in a deceleration to a repeatable, ground-referenced hover point from which rotorcraft deviations are measured. The task performance is identical to the hover MTE described in the ADS-33E [12], but the task conditions to achieve the proposed performance are distinct. While the assessing pilot performs the whole task in the original version, the adapted maneuver starts with the trainee pilot flying the vehicle, and the FI shall takeover control when the recommended flight path is violated. When the automatic inceptor decoupling is activated, the FI shall complete the transition to hover. The task is depicted in Fig. 6, and the scenario is shown in Fig. 7, including the ground references.

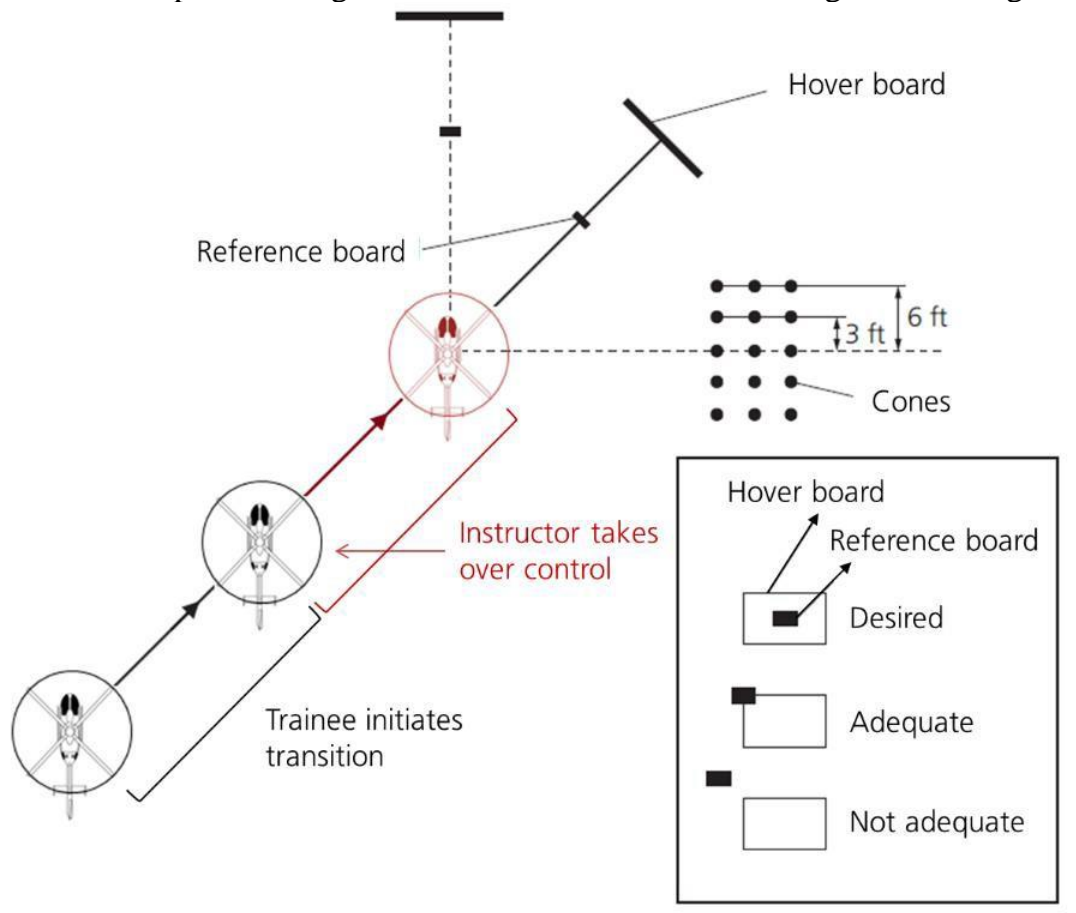

Fig. 6 Transition to hover MTE.

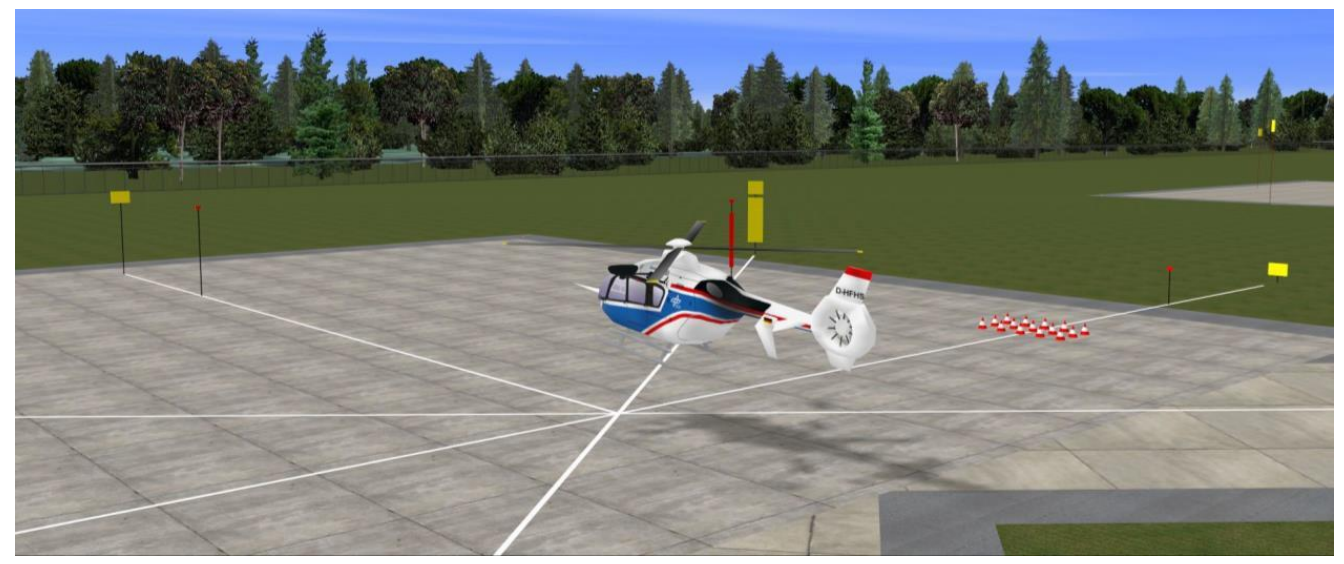

Fig. 7 Ground references of the transition to hover MTE scenario. 
According to the description of the task, the trainee pilot initiates the maneuver at a ground speed of between 6 and $10 \mathrm{knots}$, at an altitude of $15 \mathrm{ft}$. The target hover point is oriented approximately $45^{\circ}$ relative to the heading of the rotorcraft. The assessing pilot acts as the FI and uses the follow-through technique (resting hands on inceptors) to monitor the trainee's inputs. The FI counteracts the trainee pilot in case of inappropriate input regarding the ground track. When the automatic inceptor decoupling is activated, the flight instructor takes over control and completes the transition to hover. Table 2 contains the detailed performance standards for the completion of the task. The present paper focuses on the short term effects of the automatic decoupling, therefore the handling qualities analysis for the continuation of the flight (more than $10 \mathrm{~s}$ after the inceptor decoupling) is not addressed in the results' section.

Table 2 Performance standards for the transition to hover MTE

\begin{tabular}{lcc}
\hline Performance & Desired & Adequate \\
\hline Attain a stabilized hover within X s of initiation of deceleration & $5 \mathrm{~s}$ & $8 \mathrm{~s}$ \\
\hline Maintain a stabilized hover for at least & $30 \mathrm{~s}$ & $30 \mathrm{~s}$ \\
\hline Maintain the longitudinal and lateral position within $\pm X \mathrm{ft}$ of a point on the ground & $3 \mathrm{ft}$ & $6 \mathrm{ft}$ \\
\hline Maintain altitude within $\pm X \mathrm{ft}$ & $2 \mathrm{ft}$ & $4 \mathrm{ft}$ \\
\hline Maintain heading within $\pm \mathrm{X}^{\circ}$ & $5^{\circ}$ & $10^{\circ}$ \\
\hline $\begin{array}{l}\text { There shall be no objectionable oscillations in any axis either during the transition to } \\
\text { hover or the stabilized hover }\end{array}$ & $\checkmark$ & $\begin{array}{c}\text { Not } \\
\text { applicable }\end{array}$ \\
\hline
\end{tabular}

\section{F. Statistical Analysis: ROC Graph}

A statistical analysis is used to classify the severity effects of the automatic decoupling when the FT varied. This is achieved thought the receiver operating characteristics (ROC) graph, which is a technique for visualizing, organizing and selecting classifiers based on their performance [15], [16]. ROC graphs are two-dimensional graphs that illustrate the diagnostic ability of a binary classifier system as its discrimination threshold is varied. The ROC curve provides the optimum cutoff point, i.e., the threshold that makes the resulting binary prediction as close to a perfect predictor as possible [17]. The technique is based on the identification of the cutoff point value, that requires a simultaneous assessment of the proportion of subjects who are correctly diagnosed as positive (sensitivity or true positive rate) and the proportion of subjects who are correctly diagnosed as negative (specificity or true negative rate) [16]. The ROC curve is created by plotting the "1- specificity" against sensitivity at various threshold settings, as shown in Fig. 8 [18]. The perfect predictor has a single point on the graph with $100 \%$ sensitivity and $100 \%$ specificity: the upper left corner. The optimum cutoff point is defined as the point minimizing the Euclidean distance between the ROC curve and the plot point $(0,1)$, and indicates the higher discriminating capabilities among the thresholds.

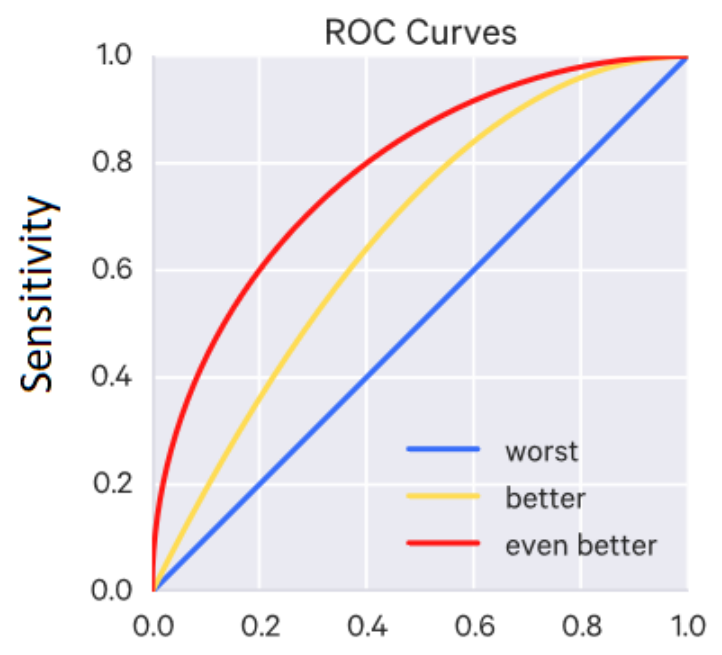

1 - Specificity

Fig. 8 ROC graph [18]. 


\section{Results}

A. Development of the Severity Envelope for the Takeover Control using the Automatic Inceptor Decoupling

The goal is to develop the severity envelope based on the transient effects (helicopter attitude and control deflection) after the automatic inceptor decoupling using different FT. The optimum FT range is the transient limit that still can be classified as minor safety severity, according to the classification of the TR (Fig. 5). The control activity was computed as root mean squared (RMS) to account for both the mean and the variance of the control deflections. The longitudinal axis was chosen because it represents the worst-case condition in terms of dynamic stability compared to the lateral axis. Six different conditions were evaluated, among which there are three force levels $(20 \mathrm{~N}, 30 \mathrm{~N}$, and $40 \mathrm{~N})$, and two force fading logic status (Counter Force on and Counter Force off), all in the modified helicopter model. A total of 90 test points were evaluated, being 15 test points in each condition.

Two main criteria are applied for the ratings: a) uniform and predictable change in the helicopter states; and b) minimum control excursions and helicopter attitude transitions. The results, shown in Fig. 9, are denoted by colors in the scatter graph. In general, the pilot rating progressively degrades with distance from the origin, which reflects an intuitively appropriate effect.

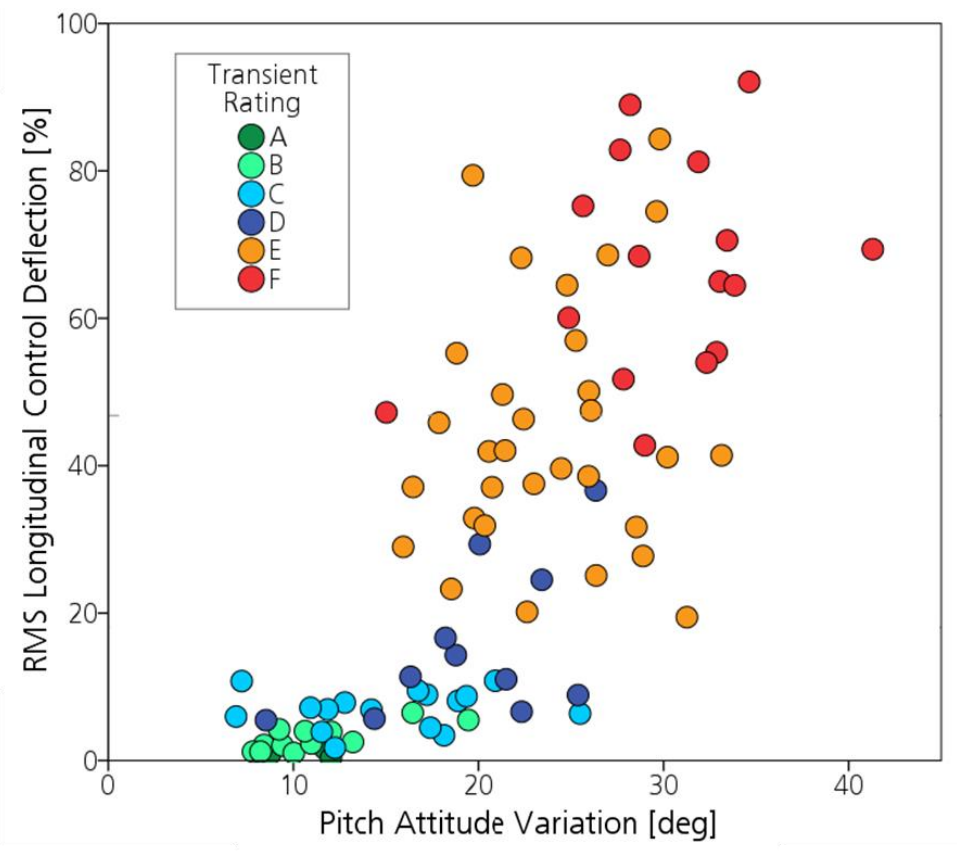

Fig. 9 Transient rating for the transition to hover task.

ROC graphs are calculated to identify the optimum cutoff point that represents the limits of the transient ratings. According to the integrated transient classification (Fig. 5) [14], the ratings A to D are associated to the minor safety severity; the rating $\mathrm{E}$ is linked to the major severity category; and ratings $\mathrm{F}$ and $\mathrm{G}$ are related to the hazardous severity category. A normality test confirmed the normal distribution of the data in each condition. The ROC graphs are presented in Fig. 10. The optimum cutoff points are the points minimizing the Euclidean distance between the ROC curve and the upper left corner, which are indicated in the Table 3. The areas under the curves in Fig. 10 are higher than or equal to 0.9 , which indicates that the models can discriminate the investigated threshold at very high precision [17].

Table 3 ROC graph values

\begin{tabular}{llcccc}
\hline Limit & Parameter & Sensitivity & 1-Specificity & $\begin{array}{c}\text { Area under } \\
\text { the curve }\end{array}$ & Value \\
\hline \multirow{2}{*}{ Transient Rating D to E } & RMS $\delta_{\mathrm{x}}$ & 0.95 & 0.00 & 0.99 & $18.05 \%$ \\
& Attitude $\theta$ & 0.83 & 0.12 & 0.92 & $19.57^{\circ}$ \\
\hline \multirow{2}{*}{ Transient Rating E to F } & RMS $\delta \mathrm{x}$ & 0.85 & 0.06 & 0.93 & $46.80 \%$ \\
& Attitude $\theta$ & 0.91 & 0.18 & 0.90 & $27.32^{\circ}$ \\
\hline
\end{tabular}




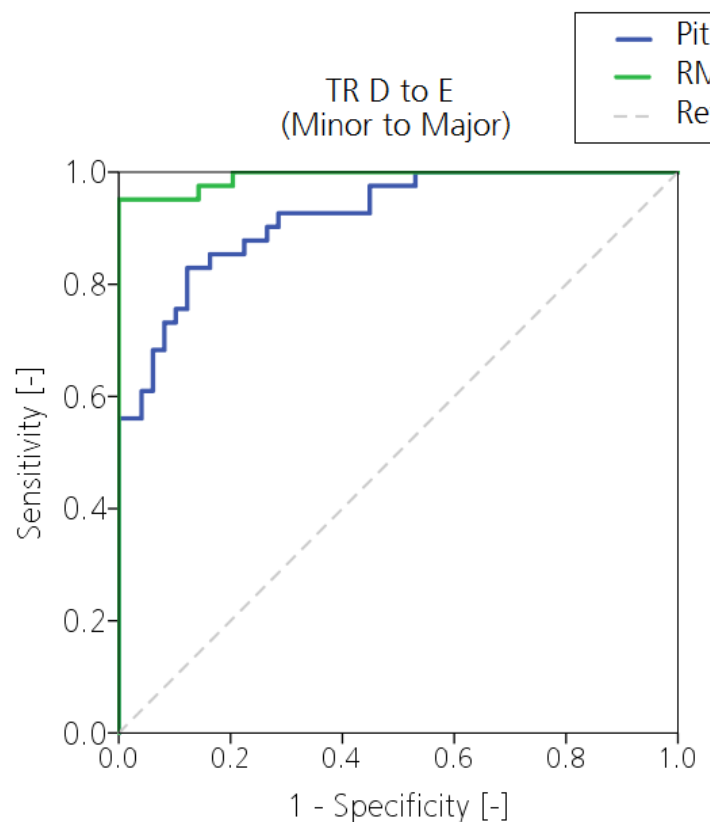

Pitch Attitude
RMS Control
Reference Line

TR E to $F$

(Major to Hazardous)

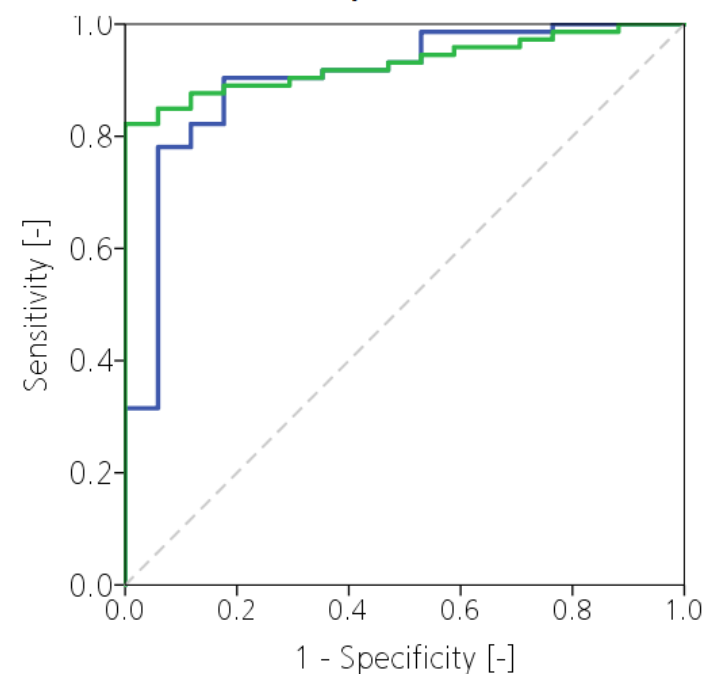

Fig. 10 ROC graphs for RMS longitudinal control deflection and pitch attitude.

The values in the last column of the Table 3 are then inserted in the graph of the pitch attitude versus RMS control, which is shown in Fig. 11. The minor severity area starts at the origin of the graph and extends transversely to the point of intersection of the optimum cutoff points regarding the transient rating $\mathrm{D}$ to $\mathrm{E}$.

Due to the rectangular shape of the thresholds, it can be concluded that the FI was tolerable to variations in attitude (abscissa axis) until $19.6^{\circ}$, but only in case of low control activity, as indicated in the ordinate axis. Similarly, the major severity area is defined by the intersection of the optimum cutoff point regarding the boundary of the transients E to F. Since no points were assigned for the transients G and H, the boundary between hazardous and catastrophic cannot be drawn.

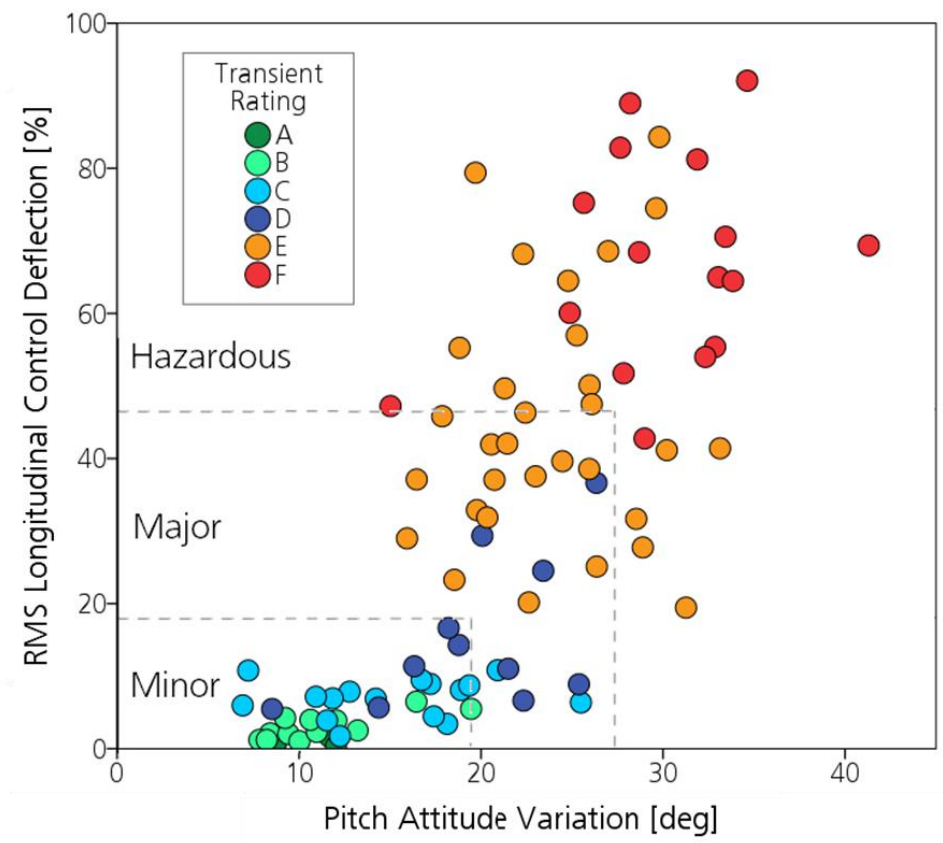

Fig. 11 Pitch attitude versus RMS control (development of severity envelope). 


\section{B. Validation of the Severity Envelope for the Takeover Control using the Automatic Inceptor Decoupling}

The validation of the severity envelope was performed by three test pilots in the task of transition to hover. The goal is to assign TR and compare with the severity envelope previously developed. The Counter Force was tested in two conditions (on and off) for three FT ( $20 \mathrm{~N}, 30 \mathrm{~N}$, and $40 \mathrm{~N})$. These six conditions were tested in both helicopter models (baseline and modified). A total of 36 test points were recorded (12 per pilot).

Fig. 12 presents the ratings assigned by the FIs regarding the transients after the automatic inceptor decoupling in takeover maneuvers. On the left side of Fig. 12, the points for the 'Counter Force off' condition are distributed along the graph in nearly linear fashion. These points can be found in all three severity areas. The test points for the 'Counter Force on' condition are situated on the right side of the same figure. The points are rather grouped in the lower part of the graph, showing that the control activity decreased compared to the 'Counter Force off' case. Also, only points in minor and major areas are found.

Based on Fig. 12, the envelope previously developed is considered valid, because only in one case there was a higher TR than the one expected by the severity area limits. The rating in this case is located close to the border of the minor area, i.e., the rightmost point in the lower graph ( $40 \mathrm{~N}$, modified helicopter, Counter Force on, TR E). Other cases of lower ratings than the ones predicted by the severity limits are not a problem in terms of safety and are considered accepted. Overall, there was a good agreement between the ratings and the envelope limits.
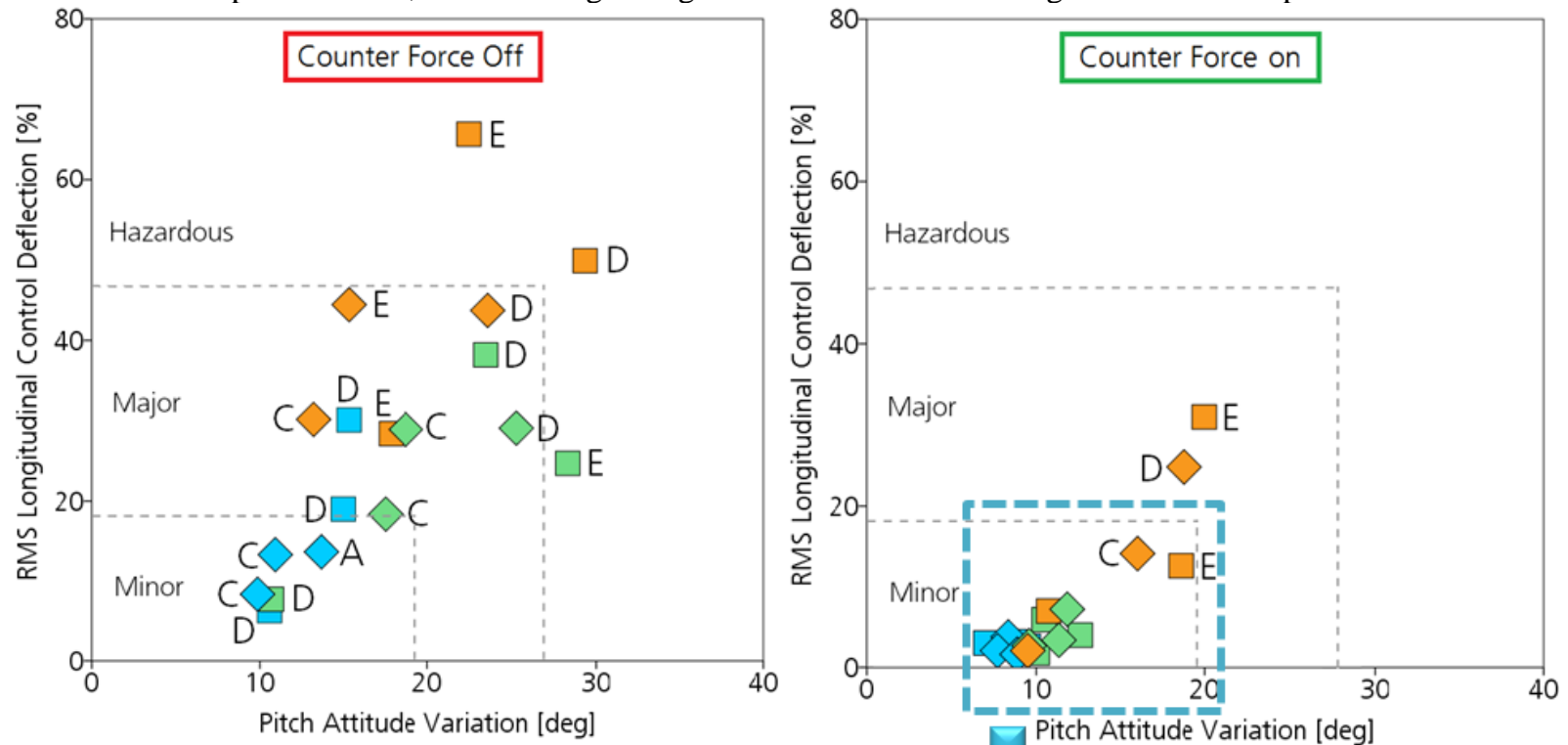

Force Threshold [N] - Helicopter Model

$20 \mathrm{~N}$ - Modified $20 \mathrm{~N}$-Baseline $30 \mathrm{~N}$ - Modified $30 \mathrm{~N}$ - Baseline $40 \mathrm{~N}$ - Modified $40 \mathrm{~N}$-Baseline

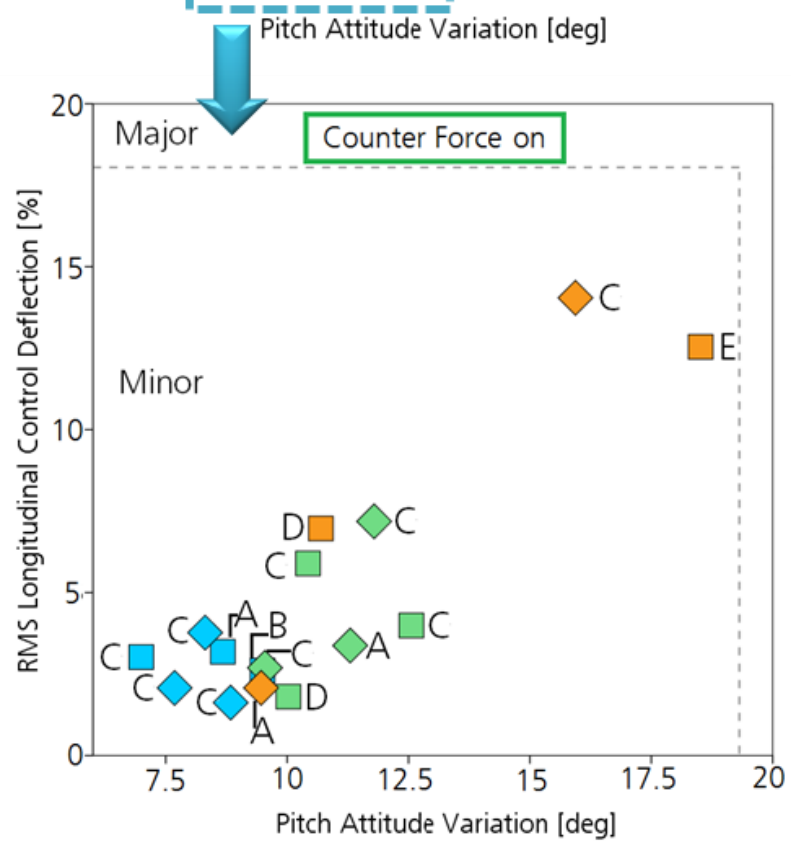

Fig. 12 Pitch attitude versus RMS control (validation of severity envelope). 


\section{Research Question 1: Maximum FT for Automatic Inceptor Decoupling}

The analysis of Fig. 12 indicates the upper FT limit in terms of controllability for automatic inceptor decoupling. The graph on the upper right side (Counter Force on) shows that the FT of $40 \mathrm{~N}$ may be excessive for the takeover maneuvers. Even when the Counter Force was activated, the test points of FT $40 \mathrm{~N}$ (orange) are spreading outside the minor area, while the test points of FT $20 \mathrm{~N}$ and $30 \mathrm{~N}$ are all concentrated in the lower middle part of this se verity category. Indeed, the FT of $40 \mathrm{~N}$ highlights the significance of the present investigation. It represents a condition in which an inceptor decoupling could induce transients that impact safety in case of control interference in low level flights; thereby the benefits of the automatic inceptor decoupling approach could be largely affected.

\section{Research Question 2: Force Fading Logic for Transient Alleviation in Automatic Inceptor Decoupling}

Regarding the effectiveness of the Counter Force to alleviate transients induced by automatic inceptor decoupling, Fig. 12 shows that the control activity and helicopter attitude variation were alleviated even for the degraded stability cases (modified helicopter model). The majority of the test points for the cases of $20 \mathrm{~N}$ and $30 \mathrm{~N}$ were classified as TR A to $\mathrm{C}$ when the force fading logic was activated.

\section{Discussion}

The severity envelope was developed based on control and attitude transient effects after the automatic inceptor decoupling. To this end, ratings for 90 test points were assigned by one test pilot according to the transient effects. ROC graphs identified the optimum cutoff points for the severity boundaries (minor/major/hazardous) by analyzing the transient ratings against two variables: RMS control deflection and attitude variation. The intersection of the cutoff points of the variables to the origin was outlined as the minor severity area, which characterized the limits of the optimum force threshold range for the automatic inceptor decoupling, according to the statistical analysis.

Three test pilots assessed the automatic decoupling system to validate the previous results. Only in one case there was a higher TR than the rating expected by the severity area limits, showing a good agreement between ratings and the envelope limits. Also, the Counter Force logic alleviated the control activity and attitude variation in the modified helicopter, resulting in acceptable transients (within the minor severity area) for the FT $20 \mathrm{~N}$ and $30 \mathrm{~N}$. The transients following the automatic decoupling, when the FT is set to $40 \mathrm{~N}$, showed significant magnitude of control and attitude (major safety severity region), thus $30 \mathrm{~N}$ is indicated as the upper boundary for the FT.

It should be highlighted that the investigation focused on the pitch axis, and the results cannot be directly transferred to other axes, inceptor types or response types. In the case of the roll axis, due to the different capabilities of the human arm and wrist, the maximum force in pitch axis is commonly indicated as 1.5 times of the maximum force in the roll axis. Thus, if the pitch FT limit is $30 \mathrm{~N}$, the roll FT limit can be specified as $20 \mathrm{~N}$.

Overall, when the Counter Force logic was activated, the automatic inceptor decoupling with a FT of $20 \mathrm{~N}$ and 30 $\mathrm{N}$ was judged by the test pilots as intuitive and effective to maintain the helicopter controllability.

\section{Conclusion}

The research focused on defining the maximum tolerable condition in the sense that HQ remain within level 2 range, which corresponds to the minor severity category. The severity envelope was positively validated by three test pilots, since there was a good agreement between the expected TR and the actual ratings assigned by the pilots. The Counter Force logic confirmed the effectiveness to reduce the control activity and attitude variation through the implementation of a force fading logic. The maximum FT for automatic inceptor decoupling is indicated as $30 \mathrm{~N}$ in pitch axis, which is the maximum FT that can be classified as a minor severity impact on flight safety.

The overall results improve the understanding of the novel inceptor decoupling functions for dual pilot helicopters. Due to safety significance of these functions for future FCS, the findings provide compelling evidence that active sidesticks can assists pilots to takeover control via automatic inceptor decoupling logics without threatening the controllability in low level flight.

\section{References}

[1] A. Taylor. The Use of Harmonic Drive Gears in Active Inceptor Systems. Harmonic Drive International Symposium Nagano, Japan, 2006.

[2] Zaichik LE, Yashin YP, Desyatnik PA, Perebatov VS, Grinev KN. Handling Quality of Aircraft Equipped with Sidesticks. 14th AIAA Aviation Technology, Integration, and Operations Conference Atlanta, US, 2014. 
[3] Summers LG, Shannon JH, White TR, Shiner RJ. SAE Technical Paper Series \# 871761: Fly-by-Wire Sidestick Controller Evaluation. SAE Aerospace Technology Conference and Exposition Long Beach, US, 1987.

[4] Field E. Handling Qualities and their Implications for Flight Deck Design. In: Harris D, editor. Human Factors for Civil Flight Deck Design. Aldershot, UK: Ashgate; 2004, p. 157-181.

[5] Field E, Harris D. A comparative survey of the utility of cross-cockpit linkages and autoflight systems' backfeed to the control inceptors of commercial aircraft. Ergonomics 1998;41(10):1462-77.

[6] Sampaio RS. Electronic Coupled Active Sidesticks in Dual Pilot Helicopters for Instructional Flights. 43rd European Rotorcraft Forum Milan, Italy, 2017.

[7] International Helicopter Safety Team. Calendar Year 2006 Report.

[8] Gerlach T, Durak U, Gotschlich J. Model Integration Workflow for Keeping Models up to Date in a Research Simulator. In: Obaidat MS, editor. 4th International Conference on Simulation and Modeling Methodologies, Technologies and Applications. Vienna, Austria: SCITEPRESS; 2014, p. 125-132.

[9] MathWorks. MATLAB and Simulink Release 2013a; Natick, US, 2013.

[10] Greiser S, von Grünhagen W. Improving System Identification Results: Combining a Physics-Based Stitched Model with Transfer Function Models obtained through Inverse Simulation. American Helicopter Society 72nd Annual Forum West Palm Beach, US, 2016.

[11] von Grünhagen W, Müllhäuser M, Abildgaard M, Lantzsch R. Active Inceptors in FHS for Pilot Assistance Systems. 36th European Rotorcraft Forum Paris, France, 2010.

[12] United States Army Aviation, Missile Command Aviation Engineering Directorate. Rotorcraft Aeronautical Design Standard Performance Specification, Handling Qualities Requirements for Military; Redstone Arsenal, US, 2000.

[13] Weakley JM, Kleinhesselink KM, Mason D, and Mitchell D. Simulation Evaluation of V-22 Degraded Mode Flying Qualities. American Helicopter Society 59th Annual Forum Phoenix, US, 2003.

[14] Padfield G. Helicopter Flight Dynamics, Second Edition. Washington DC, US: American Institute of Aeronautics and Astronautics, Inc; 2007.

[15] Fawcett T. An introduction to ROC analysis. Pattern Recognition Letters 2006;27(8):861-74.

[16] Pepe MS. The statistical evaluation of medical tests for classification and prediction. Oxford, UK: Oxford University Press; 2004.

[17] Gönen M. Analyzing Receiver Operating Characteristic Curves with SAS. Cary, NC: SAS Pub; 2007.

[18] Tseng F. Evaluating classification models: Area under the curve (AUC). [April 18, 2018]; Available from: https://frnsys.com/ai_notes/machine_learning/model_selection.html. 